\title{
BMJ Open Making a balanced plate for pregnant women to improve birthweight of infants: a study protocol for a cluster randomised controlled trial in rural Bangladesh
}

\author{
Morseda Chowdhury, ${ }^{1,2}$ Camille Raynes-Greenow, ${ }^{1}$ Ashraful Alam, ${ }^{1}$ \\ Michael J Dibley ${ }^{1}$
}

To cite: Chowdhury M, Raynes-Greenow C, Alam A, et al. Making a balanced plate for pregnant women to improve birthweight of infants: a study protocol for a cluster randomised controlled trial in rural Bangladesh. BMJ Open 2017;7:e015393. doi:10.1136/ bmjopen-2016-015393

- Prepublication history and additional material for this paper are available online. To view these files please visit the journal online (http://dx.doi. org/10.1136/bmjopen-2016015393).

Received 1 December 2016 Revised 18 April 2017 Accepted 21 April 2017

\section{CrossMark}

${ }^{1}$ Sydney School of Public Health, The University of Sydney,

Sydney, Australia

${ }^{2}$ Health Nutrition and Population Programme, BRAC, Bangladesh

Correspondence to Dr Morseda Chowdhury; morsheda.c@brac.net

\section{ABSTRACT}

Objectives Low birthweight significantly contributes to neonatal mortality, morbidities and psychosocial debilities throughout the course of life. A large proportion of infants (36-55\%) in Bangladesh is born with low birthweight. Nutritional status of women during pregnancy is critical for optimal growth and development of the fetus. Nutrition education has been found to improve maternal nutritional status. Our study aims to determine whether nutrition education with a practical demonstration during pregnancy is an effective intervention for improving the birthweight of infants compared with standard nutrition education only.

Methods and analysis We will conduct a community-based cluster randomised controlled trial in one rural district of Bangladesh. Treatments will be allocated evenly between the study clusters $(n=36)$. Participants in the intervention clusters receive 'balanced plate nutrition education' with a practical demonstration from community health workers 4-7 times throughout their entire pregnancy, starting from the first trimester. The control clusters will receive standard nutrition education delivered by public and other healthcare providers as per ongoing antenatal care protocol. Our sample size would be 900 pregnant women to determine $100 \mathrm{~g}$ differences in mean birthweight, considering 5\% type 1 error, $80 \%$ power and an intra-cluster correlation coefficient of 0.03 . The primary outcome of the trial is birthweight of the infants and the secondary outcomes include daily caloric intake and dietary diversity score among the pregnant women. Outcomes will be measured at enrolment, third to ninth month of gestation (monthly) and at delivery. Community health workers blinded to the study hypothesis will collect all data.

Ethics and dissemination The study was approved by the James P Grant School of Public Health, BRAC University Ethical Review Committee, Dhaka, Bangladesh. We will communicate the final results to relevant research and public health groups and publish research papers in peer-reviewed journals. Trial registration number ACTRN12616000080426.
Strengths and limitations of this study

- This study may contribute to limited evidence regarding maternal nutrition education strategies to improve birthweight of infants.

- Identifying effective approaches in nutrition education is likely to contribute to reducing the burden of maternal malnutrition and poor birth outcomes.

- This replicable behaviour change communication approach can be scaled up and sustained through existing health systems with minimal investments.

- Prior piloting and adaptation are required to replicate this community-based intervention in other settings.

- The study is localised in one district of Bangladesh, so the results might not be representative or generalisable to all parts of Bangladesh or other countries.

\section{INTRODUCTION}

Background and rationale

Globally more than 20 million infants are born with low birthweight (LBW) $(<2500 \mathrm{~g})$ every year, $96 \%$ in low and middle-income countries. ${ }^{1}$ Neonates weighing less than 2500 $\mathrm{g}$ are approximately three times more likely to die compared with those weighing 2500 $\mathrm{g}$ or more at birth, and the risk increases as birth- weight decreases. LBW indirectly accounts for $60 \%$ of the deaths among neonates and more than 13 million disability adjusted life years among children aged less than five years. ${ }^{2}$ LBW significantly impacts on health throughout the life course; in childhood it causes hyperactivity and inattention, emotional and behavioural problems and poor educational attainment, ${ }^{3}$ while in adulthood it leads to the development of chronic diseases ${ }^{4}$ and lower economic productivity. ${ }^{5}$ 
Intrauterine growth restriction (IUGR) is the probable cause of LBW in babies born at term ( $\geq 37$ weeks of gestation $)^{2}$; this may be partly due to the impairment of the placental development and function in the presence of maternal undernutrition. ${ }^{6}$ There is strong evidence that poor maternal nutrition during pregnancy leads to intrauterine growth restriction and thus LBW. ${ }^{7-9}$ Thirteen percent of Bangladeshi women of reproductive age (15-49 years) are short statured (height $<145 \mathrm{~cm}$ ) and $19 \%$ are thin (body mass index $<18.5 \mathrm{~kg} / \mathrm{m}^{2}$ ), ${ }^{10}$ which indicates that these women enter pregnancy with chronic undernutrition. Moreover, in Bangladesh during pregnancy women consume far less than the recommended dietary energy. Alam and colleagues found that the average daily energy intake among Bangladeshi women in late pregnancy was $1464 \pm 416 \mathrm{kcal}$ per day, much lower than the recommendation of $\sim 2500 \mathrm{kcal}^{11}{ }^{11}$ Among women from low-income families, diets lack protein and micronutrients. ${ }^{7}$ Dietary diversity, which is believed to be strongly associated with nutrient adequacy, was 4.5 (out of nine food subgroups) in women's regular diet in rural Bangladesh. ${ }^{8}$ This nutritional deprivation during pregnancy may impair placental development, leading to reduced nutrient transfer to the fetus and ultimately to IUGR. ${ }^{9}$ A longitudinal study conducted in Iran between 2009 and 2010 found that energy and protein intake was associated with the birthweight of infants. Pregnant women who consumed $1794 \pm 54 \mathrm{kcal}$ per day gave birth to neonates with a mean birthweight of $2600 \mathrm{~g}$, whereas pregnant women who consumed $2698 \pm 107 \mathrm{kcal}$ gave birth to neonates with a mean birth- weight of $3600 \mathrm{~g}$. Similarly, an increase in protein intake from $38.7 \pm 4.7 \mathrm{~g}$ to $72.8 \pm 7.4$ $\mathrm{g}$ per day was associated with an increased birthweight from $2500 \mathrm{~g}$ to $3400 \mathrm{~g} .{ }^{12}$

Antenatal nutrition education has been found to improve dietary behaviour and significantly increase total energy intake (from 2269 to $2431 \mathrm{kcal}$ per day, $\mathrm{p}<0.05$ ) among low-income pregnant women. ${ }^{13}$ A systematic review examined the effect of antenatal nutrition education on birthweight of infants and found a significant increase in birthweight by $105 \mathrm{~g}$. However, the stratified analysis showed that the effect was significant only for high-income countries and when nutrition education was provided with nutritional supplementation..$^{14}$ A Cochrane review reported a significant increase in birthweight among undernourished women who received nutrition education (difference in mean $490 \mathrm{~g}$; 95\% CI 428-552 g), while no significant increase was observed for adequately nourished women. ${ }^{15}$ These findings are based on studies with some methodological weaknesses including small sample size or few events and quasi-experimental studies or randomised controlled trials with design or analysis limitations.

Bangladesh has had a large-scale community-based nutrition programme since 1995 and mainstreamed antenatal nutrition education and supplementation programmes since $2010 .{ }^{16}$ Despite these programmes, the LBW rate still remains a public health concern, estimated at $36-55 \% .{ }^{17-19}$ In this context, innovative interventions are needed to accelerate reductions in LBW at the population level to achieve the Sustainable Development Goal targets of nutrition by $2030 .{ }^{20}$ Shifting from conventional nutrition education and food supplementation to more effective enhanced nutrition education could be one of the potential sustainable ways of improving dietary behaviour during pregnancy and to increase birthweight at the population level.

\section{Study objectives and hypothesis}

We have designed a nutrition education intervention that actively teaches and empowers pregnant women to prepare their own balanced plate of food that includes an appropriate selection of items with maximisation of diversity and adequate portion size from foods readily available in their kitchen. The objective of the study is to compare the impact of this 'balanced plate nutrition education' approach with the standard nutrition education programme for pregnant women on their dietary behaviour and birthweight of their infants.

Our primary hypothesis is that women who receive 'balanced plate nutrition education' during pregnancy will deliver babies $100 \mathrm{~g}$ heavier than those who receive standard nutrition education. Secondary hypotheses are that 'balanced plate nutrition education' will increase daily caloric intake by $300 \mathrm{kcal}$ and increase the dietary diversity score to at least five (out of nine food subgroups with minimum consumption of $15 \mathrm{~g}$ from each group) among pregnant women compared with standard nutrition education.

\section{METHODS AND ANALYSIS \\ Study design}

We will conduct a two-arm parallel community-based cluster randomised controlled trial with pregnant women. Women allocated to the intervention arm will receive the 'balanced plate nutrition education' with practical demonstrations and women in the control arm will receive standard nutrition education (figure 1). This is an effectiveness trial to be conducted through the existing health programme of BRAC (an NGO formerly known as Bangladesh Rural Advancement Committee or Building Resources Across Communities), a nationwide community-based healthcare intervention.

\section{Formative research}

We will conduct a food attributes exercise applying the ProPAN methodology. ${ }^{21}$ In this regard, a group of qualitative interviews will take place with key target populations such as pregnant women, influential family members (eg., husbands, mothers and mother-in-laws of the pregnant women) to generate information about key foods taken during pregnancy, positive and negative characteristics attributed to key foods, and conditions and changes required for pregnant women to consume nutrient-rich foods that are not currently taken or 


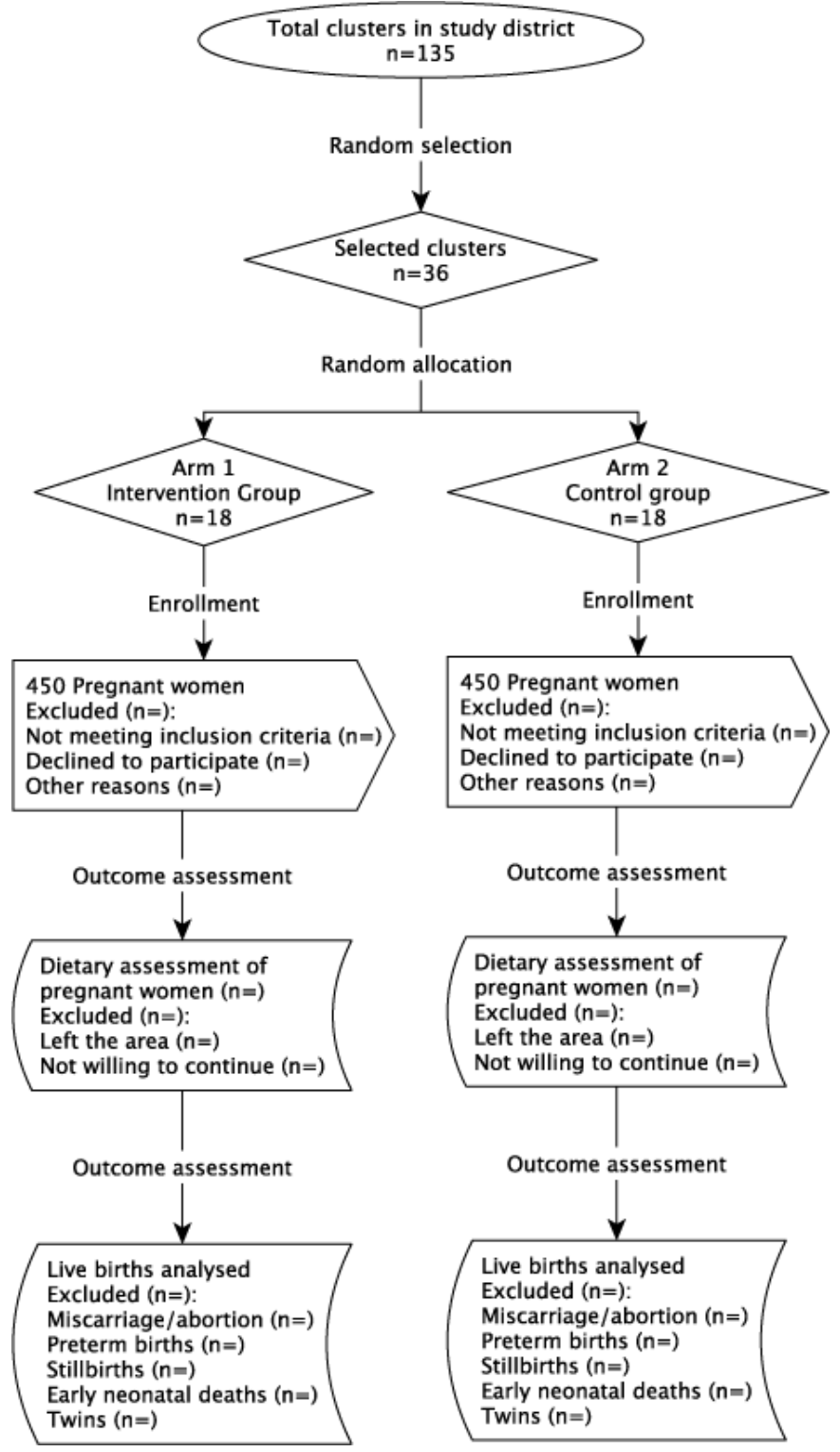

Figure 1 Study design

inadequately taken. These data will inform the design of the 'balanced plate nutrition education' intervention, which will then be pilot tested to assess its acceptability to the community and the feasibility of implementation prior to the commencement of the actual trial. We will conduct focus group discussions with pregnant women; their husbands, mothers and mother-in-laws; and Shasthya Kormis (community health workers of BRAC who are the major antenatal care providers in the respective community) in two different sites of the study area. The data will assist with understanding the cultural compatibility of the proposed approach along with identifying local norms, beliefs, priorities and any cultural food restrictions and prescriptions during pregnancy. Information about the availability of foods in different seasons will also be considered. The final messages will be developed, refining the menu by incorporating the results of the pilot trial on local preferences, acceptability and seasonal variation.

\section{Study setting}

The proposed research will take place in the villages of Sherpur district, situated in the northern part of Bangladesh (figure 2). The total population of the district is approximately 1.4 million. The economy of Sherpur is mainly agriculture-based with $60 \%$ of the households engaged in farming. The adult literacy rate is $37.9 \%$ (male $40.2 \%$ and female $35.7 \%$ ), ${ }^{22}$ much lower than the national average of $56.1 \% .{ }^{23}$ Healthcare services such as maternity care are mostly provided by the public providers through healthcare facilities and outreach centres.

BRAC implements a community-based maternal, neonatal and child health programme in the study area, characterised by a prospective pregnancy surveillance system, home-based antenatal care (ANC) and other reproductive, neonatal and child healthcare. The trial uses the established pregnancy surveillance and homebased ANC system of BRAC. There are $~ 346$ health volunteers (called Shasthya Shebika) who pay home visits to women of reproductive age and detect delayed or missed menstrual cycles. Women missing a menstrual cycle in the previous sixweeks are screened for possible pregnancy using a urine test and, if the pregnancy is confirmed, start receiving ANC from the Shasthya Kormis (SK).

\section{Study clusters}

We have chosen clusters as the unit of randomisation instead of individuals for greater logistical convenience and to prevent contamination of the intervention. The unit of randomisation in our study is the population $(\sim 10000)$ served by $S K$ with a defined geographical area consisting of approximately five villages. The entire study district is divided into 135 exclusive clusters. Clustering will help minimise sharing of nutrition messages among pregnant women, which would occur if the intervention and control were administered among women from the same village or neighbouring villages. Clusters will be eligible for inclusion in the trial if the corresponding SKs have work experience for at least six months in the maternal, neonatal and child health programme in the specific region. Clusters will be excluded if there is any maternal nutrition intervention already in place.

\section{Study population}

The study population comprises pregnant women permanently residing in the study area. Permanent residency is defined by living in the study area for a minimum of six consecutive months preceding the recruitment.

\section{Inclusion and exclusion criteria}

We will consider women eligible for recruitment if they are:

- married and of reproductive age (15-49 years)

- pregnant with a duration of gestation of 7-12 weeks

- permanent residents of the study area. 


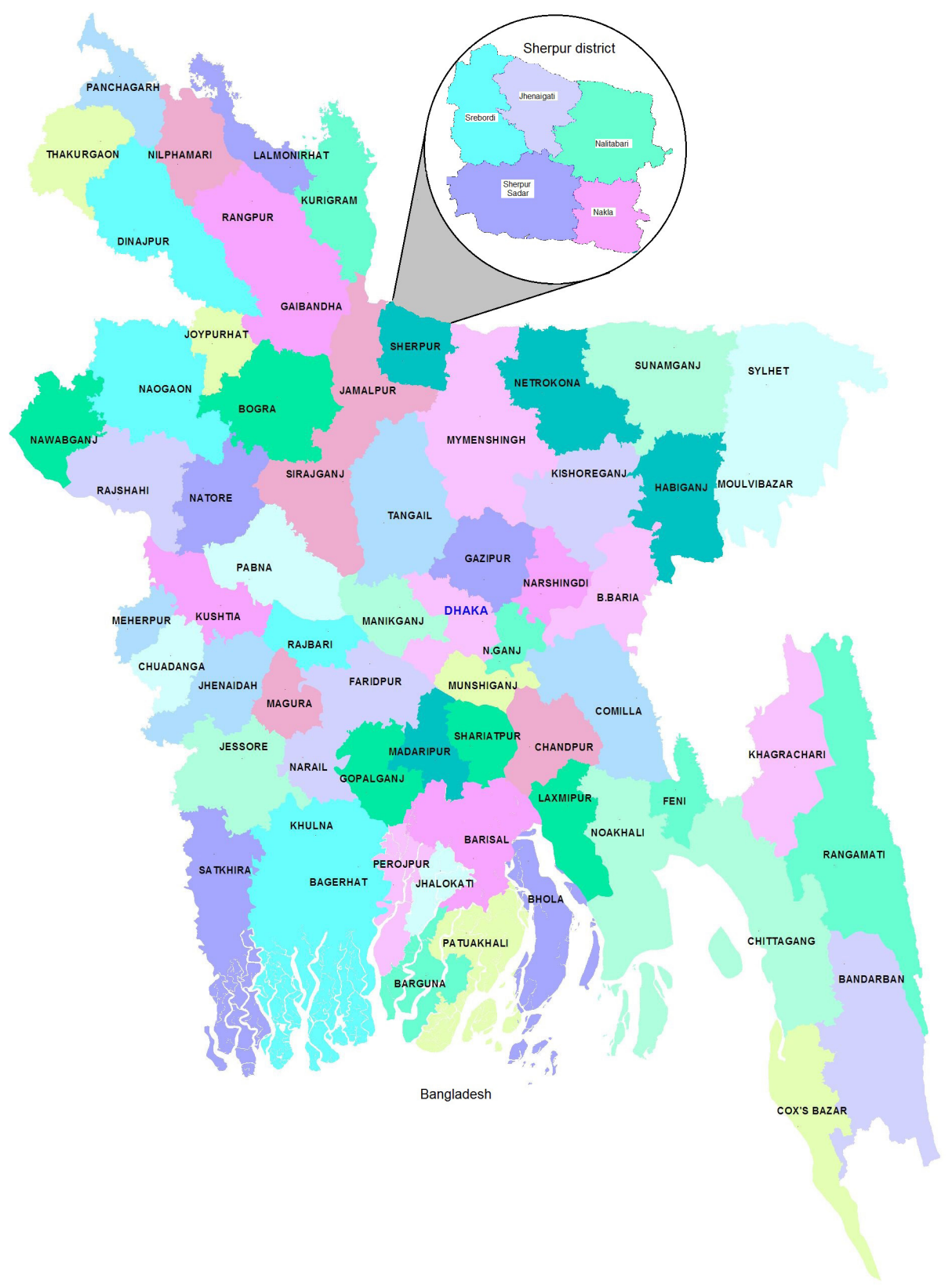

Figure 2 Study site

We will exclude women who have:

- planned to deliver outside the study area

- been diagnosed with any chronic diseases, such as diabetes, hypertension and other diseases that may impact on their ability to participate in the trial.

\section{Randomisation}

Random selection of clusters

Sherpur district is divided into five sub-districts: Jhenaigati, Nakla, Nalitabari, Sherpur Sadar and Sreebardi containing 16, 19, 25, 49 and 26 clusters, respectively. We will use proportionate stratified sampling method to obtain 4, $5,7,13$ and 7 clusters, respectively, from each of the five sub-districts proportionate $(36 / 135)$ to their population size. For the first sub-district (Jhenaigati), we will select four clusters from a list of the clusters in that area using random numbers generated by a computer program in MS Excel spreadsheet (using the RANDOM function), and apply the same method to the rest of the sub-districts until we obtain the 36 clusters. With this technique, we will be able to capture geographical variations across the district.

Allocation sequence and concealment

Once we have prepared the list of clusters, we will conduct a lottery to assign treatments between the two groups (intervention (group A) and control (group B)) in 1:1 ratio. We will write each cluster number onto 36 pieces of paper and put them into five different jars, one jar for each sub-district, based on the cluster selection made in the first step. The treatment sequence will alternate between A (intervention) and B (control), starting with A. One volunteer (not 
involved with the study) will pick the papers blindfolded. For the first sub-district, there will be four pieces of paper and the treatment assignment sequence will be $\mathrm{ABAB}$, which means the first number picked will go to treatment $\mathrm{A}$ and the next to treatment $\mathrm{B}$ and so on. For the second sub-district, the sequence would be ABABA, starting with where the first one ended. Subsequently, the sequence we will follow for the third, fourth and fifth sub-districts are BABABAB, ABABABABABABA and BABABAB.

Implementation

BRAC staff from the headquarters along with the Principal Investigator will select the clusters as described above, while BRAC local staff (at the study site) will conduct the lotteries to assign the treatments in the presence of other staff in the office. After obtaining consent from the SKs to take part in the study, the lists will be provided to the training unit to ensure appropriate training of the two groups. If any $S K$ decides to withdraw from the study, she will be replaced by another $S K$ randomly selected from the remaining $S K s$ in the list.

\section{Enrolment}

Once clusters are finalised, SKs will start enrolling study participants, consented eligible pregnant women, from the study sites. Each $S K$ has been given a minimum target to enrol 10 pregnant women per month until the individual target (25) is reached. Regular pregnancy identification system of BRAC will be used to reach pregnant women and approach them for obtaining the consent. The long-term involvement of $S K$ s with the community enabled them to gain people's trust, which will help maximise enrolment and obtain the desired sample size within threemonths. However, if the target is not reached, the enrolment period will be extended with proper justification. Actual enrolment started on 1 October 2016 and will continue until 31 December 2016. The details of the enrolment schedule are shown in table 1.

Table 1 Time schedule of enrolment, intervention, assessment and process evaluation

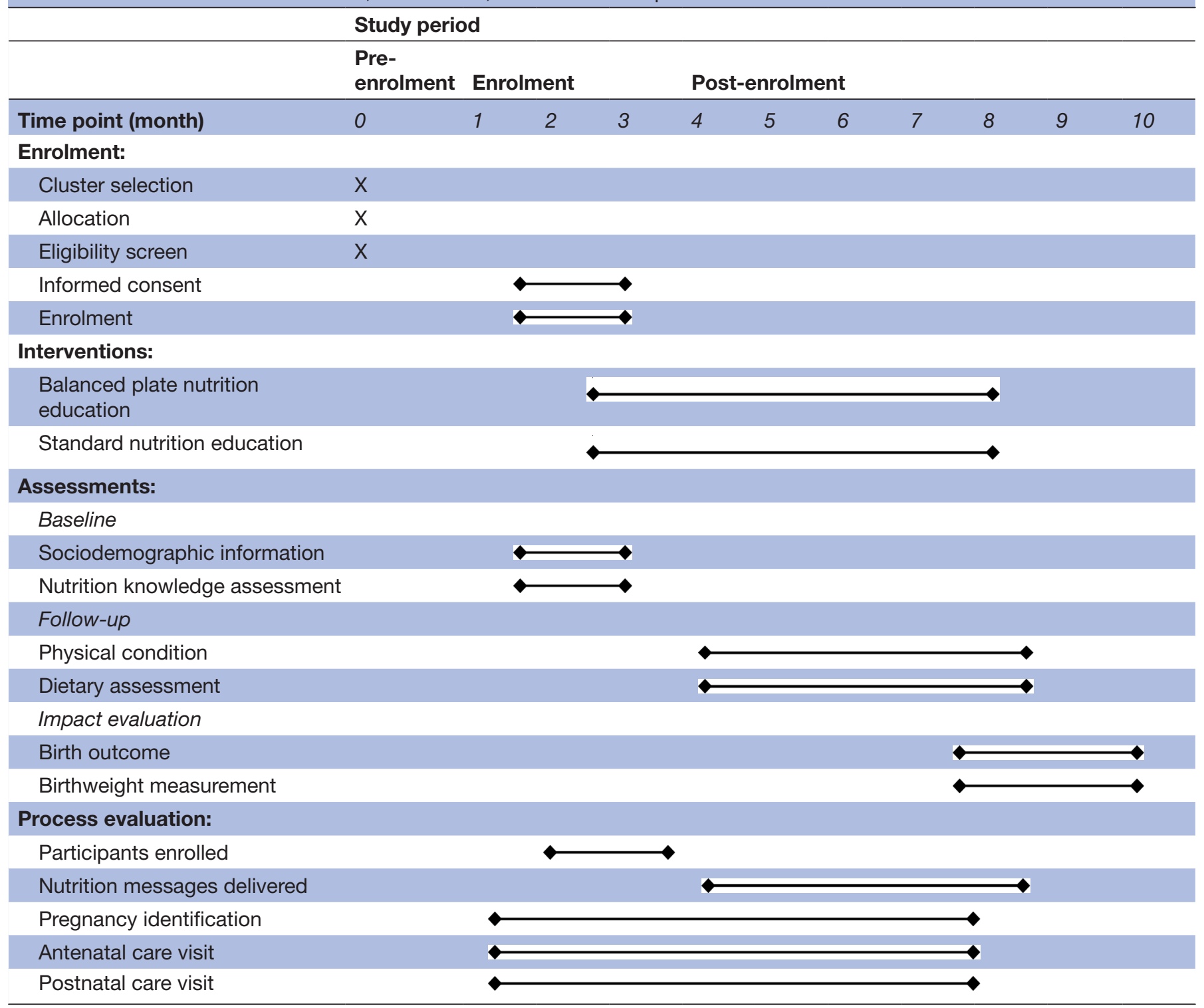




\section{Box Core contents of nutritional messages}

1. Eat five times a day, three major meals and two snacks.

2. Eat 1.5 dishes of rice or 3 pieces (medium sized) of chapaties, 1 dish of vegetables, 1 egg or 1 dish of thick lentil in the morning.

3. Eat 1 piece or 1 dish of seasonal fruit(s) and 1 dish of milk product(s) as morning snack.

4. Eat 3 dishes of rice, 1 dish of vegetables, 1 dish of thick lentil and 1 piece of fish or meat or egg at lunch.

5. Eat 1 glass of milk, 1 dish of puffed rice mixed with molasses and 1 piece or 1 dish of seasonal fruit(s) in the evening snack.

6. Eat 2 dishes of rice, 1.5 dishes of vegetables, 1 dish of thick lentil, 1 piece of fish or meat or egg and 1 glass of milk or 0.5 dish of curd at dinner.

7. Drink at least 8 glasses of water.

8. Cook with oil.

\section{Blinding (masking)}

Allocation concealment is not possible due to the nature of the intervention. The participants, as well as the $S K$ s, are aware of the intervention. However, both the participants and the $S K$ s will be blinded to the study hypothesis. We will use objective and reliable outcomes, such as birthweight and food consumption, which are less prone to ascertainment bias. Finally, we will blind the data analysts by labelling the groups with non-identifying terms (e.g; A and B) until the final analysis.

\section{Intervention}

The study intervention, 'balanced plate nutrition education', is a method of teaching pregnant women to prepare properly balanced meals. The education session has two parts: one-to-one nutrition-specific counseling and practical demonstration. For counselling, SKs will use written and pictorial menus. The core contents of the counselling are included in box. The nutritional messages are developed with reference to the current Bangladeshi recommendations, ${ }^{24}$ which incorporates the following: (a) intake of food-yielding at least $2500 \mathrm{kcal}$ energy per day; (b) consumption of diversified food; (c) inclusion of animal sourced food in the diet, at least two servings per day; and (d) eating at least five times a day (five different plates for five meals). Rather than endorsing new or expensive foods, indigenous foods rich in iron, calcium and vitamins will be promoted in the study. Considering the high price and low consumption of protein-rich foods in general, both from the plant (ie, lentils) and the animal (ie, meat, fish, egg and milk) sources, we will advise the cheapest options for protein from animal source such as fish grown in local farms. There are varieties of inexpensive fish available in the market, such as tilapia, certain catfish (e.g., pangas) and carp (e.g., silver carp). We will motivate the pregnant women to consume an adequate amount of these fish by making them realise that these are equally as nutritious as other costly protein-rich foods. Five different menus will be developed for five daily meals yielding collectively $2500 \mathrm{kcal}$ energy and essential micro- and macronutrients for the day (table 2). A pictorial menu (figure 3 ) and a pictorial food
Table 2 Five-meal menu for pregnant women (English)

\begin{tabular}{|c|c|c|}
\hline Meal & Food & $\begin{array}{l}\text { Quantity (one } \\
\text { dish }=250 \mathrm{~mL} \text { ) }\end{array}$ \\
\hline \multirow[t]{7}{*}{ Breakfast } & Rice & 1.5 dishes \\
\hline & or & \\
\hline & Chapati (medium size) & 3 pieces \\
\hline & Vegetables & 1 dish \\
\hline & Egg & 1 \\
\hline & or & \\
\hline & Lentil (thick) & 1 dish \\
\hline \multirow[t]{2}{*}{$\begin{array}{l}\text { Mid-morning } \\
\text { snack }\end{array}$} & Seasonal fruit(s) & 1 piece/dish \\
\hline & Milk product(s) & 1 dish \\
\hline \multirow[t]{4}{*}{ Lunch } & Rice & 3 dishes \\
\hline & Lentil (thick) & 1 dish \\
\hline & Leafy/non-leafy vegetables & 1.5 dishes \\
\hline & Meat/fish/egg & 1 piece \\
\hline \multirow[t]{3}{*}{$\begin{array}{l}\text { Afternoon } \\
\text { snack }\end{array}$} & Milk & 1 glass \\
\hline & Seasonal fruit(s) & 1 piece/dish \\
\hline & $\begin{array}{l}\text { Puffed rice with molasses } \\
\text { or } \\
\text { Biscuits }\end{array}$ & $\begin{array}{l}1 \text { dish } \\
1 \text { dish }\end{array}$ \\
\hline \multirow[t]{6}{*}{ Dinner } & Rice & 2 dishes \\
\hline & Lentil (thick) & 1 dish \\
\hline & Leafy/non-leafy vegetables & 1.5 dishes \\
\hline & Meat/fish/egg & 1 piece \\
\hline & $\begin{array}{l}\text { Milk } \\
\text { or }\end{array}$ & 1 glass \\
\hline & Curd & 0.5 dish \\
\hline
\end{tabular}

chart (figure 4) in the local language will be provided to all women, who are mostly illiterate or semi-literate.

Practical demonstration refers to exhibiting the meal preparation in front of the pregnant women and their family members in a participatory way. For this, participant women will be encouraged to share food from their own. If the available food items are not sufficient to make a balanced meal, then the neighbours will be asked to contribute. Sharing food between households is common and culturally acceptable in rural Bangladesh. SKs will measure portion sizes for carbohydrate, protein and vegetable groups using a local utensil (250 $\mathrm{mL}$ size dish) and display the assembled meal on a regular dinner plate. From this visual presentation, pregnant women will be able to actively identify the food groups where they should make a change. Pregnant women are then encouraged to make their own plate for each meal following the given menu. The $S K$ s will invite the key influential family members to join the balanced plate preparation demonstration and the counselling sessions. The mother-in-law and/or mother of the pregnant woman (depending on where the 


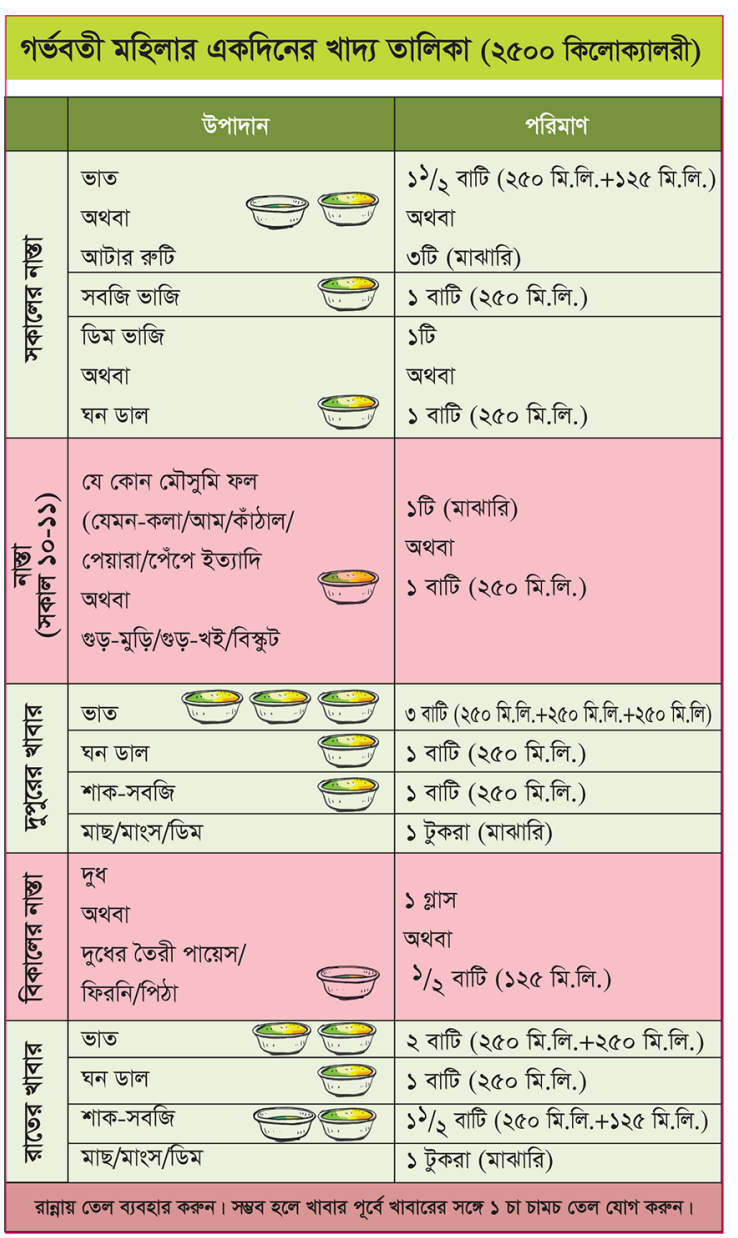

Figure 3 Five-meal menu for pregnant woman (Bengali).

women stay at the time of counselling/demonstration) and her husband (if he is available) will be invited. In the absence of the mother-in-law or mother, an influential senior female member will be invited. In addition to providing counselling on the maternal nutrition and balanced diet, the $S K$ s will motivate these family members to provide support to the pregnant women for their adherence to the nutritional messages. We expect that their active support will create an enabling environment for the pregnant women to practise improved diet.

The intervention will commence in the first trimester (ideally before 12 weeks of gestation) and will continue every month until birth. Each pregnant woman will have 4-7 sessions throughout her entire pregnancy. The sessions will last for approximately $30-45 \mathrm{~min}$.

\section{Control intervention}

Government health facilities provide nutrition education, following a national standard protocol, to all pregnant women seeking ANC. Pregnant women from both the control and the intervention groups will continue to have access to this service. This education is one-to-one advice, delivered by doctors or Family Welfare Visitors. The content usually includes the following advices: (a) taking extra food; (b) consumption of meat, fish, liver, egg, milk/milk products, lentils, colourful vegetables, fruits and oil; and (c) taking ironfolic acid (60 $\mathrm{mg}$ iron and $40 \mathrm{mg}$ folic acid) and calcium $(500 \mathrm{mg})$ supplements. The control group has the same frequency of contact with the $S K$ s as the intervention group and receives antenatal care except for the 'balanced plate nutrition education'.

\section{Outcomes}

\section{Primary outcome}

The primary outcome of this study is the birthweight of the newborn infants. Community health volunteers notify births in the community within 24 hours. SKs visit the mother and baby, and conduct a postnatal check-up including weighing the baby. Birthweight is defined as the weight of an infant just after birth. Birthweight less than $2500 \mathrm{~g}$ is considered as low birthweight.

\section{Secondary outcomes}

The secondary outcome is the maternal dietary behaviour, which includes daily caloric intake and dietary diversity score. SKs will conduct dietary assessments with a semi-structured questionnaire, developed based on the list of foods used in the Food and Nutrition Technical Assistance II Project (FANTA-2) ${ }^{8}$ This tool captures detailed information about all foods, beverages and dietary supplements consumed in the past 24 hours with quantity, from midnight to midnight the previous day. Prompts, such as a $250 \mathrm{~mL}$ size dish, will be used to improve estimation of portion size. We will estimate the approximate weight in grams using the conversion table from the Food Composition Table for Bangladesh (INFS 2013) to calculate the equivalent weight of the raw food and attainable calories. ${ }^{25}$ We will combine all consumed food items in 10 groups as: (1) grains, white roots and tubers; (2) pulses (beans, peas and lentils); (3) nuts and seeds; (4) dairy; (5) meat, poultry and fish; (6) eggs; (7) dark green leafy vegetables; (8) other vitamin A-rich fruits and vegetables; (9) other vegetables; and (10) other fruits. Minimum dietary diversity, a dichotomous indicator, will be developed for referring higher micronutrient adequacy and diet quality. ${ }^{7}$

\section{Other study parameters}

Other study parameters include age, education and occupation of mother; age, education and occupation of husband; religion; family income; living children; previous pregnancy loss; gestational age; tetanus injection; ANC visit; and other healthcare services used.

\section{Sample size calculation}

A published randomised controlled trial in rural Bangladesh reported a mean \pm SD birthweight of $2531 \pm 415$ g. ${ }^{19}$ Holding this as a reference, we expect a $100 \mathrm{~g}$ increase in birthweight over the study period in the intervention group, resulting in a mean expected birthweight of 2631 g. Using standard sample size calculation formulae, ${ }^{26}$ the estimated sample size will be 720 live births (from 36 clusters) to determine a $100 \mathrm{~g}$ difference in mean birthweight, assuming $5 \%$ type 1 error, $80 \%$ power and a 0.03 


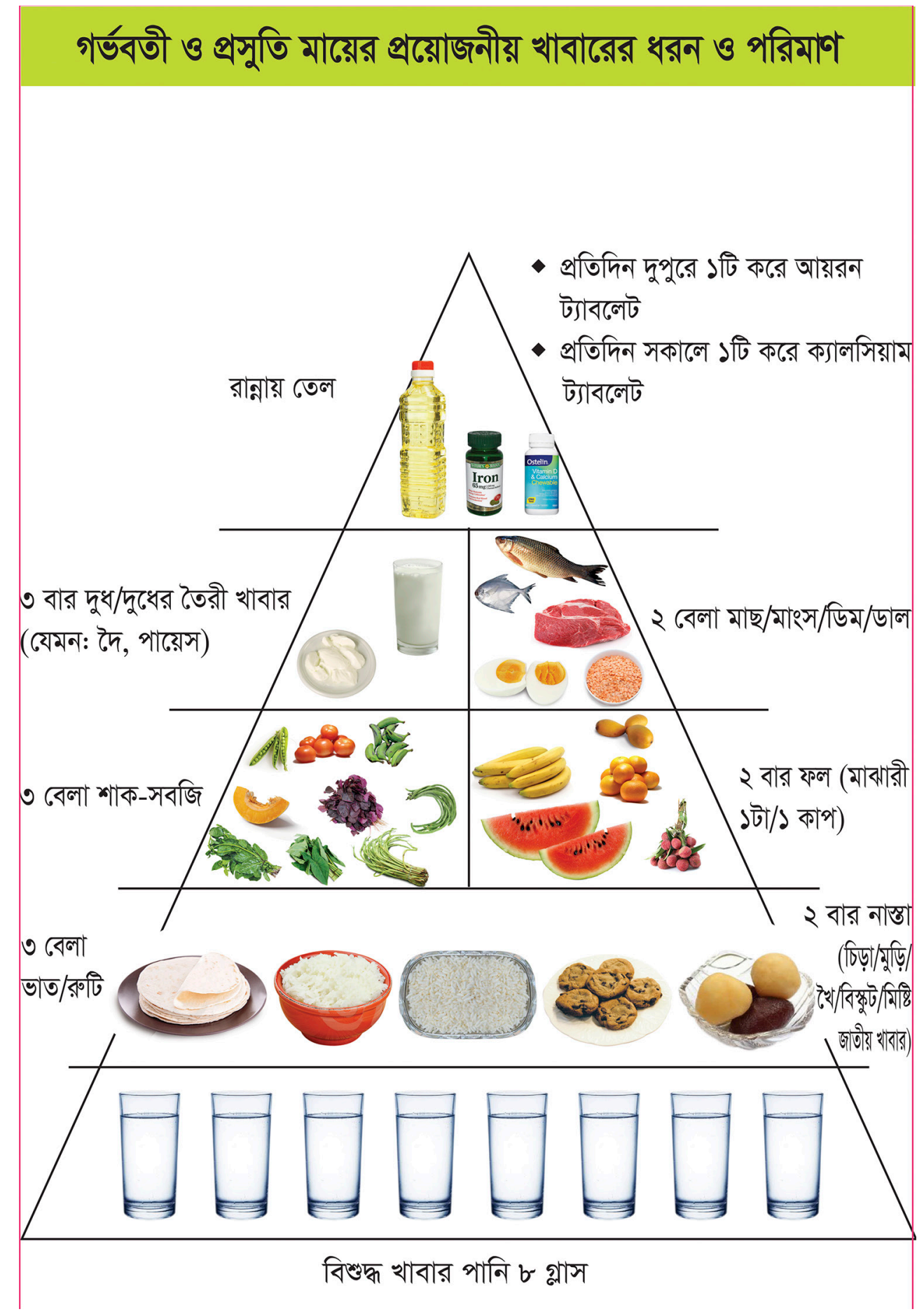

Figure 4 Pictorial food chart for pregnant women (bengali)

intra-cluster correlation coefficient (ICC) (based on a published ICC for birthweight from a trial conducted in rural China). ${ }^{27}$ Inflating the sample by $5 \%$ for non-response, $10 \%$ for pregnancy loss and $10 \%$ for delivery outside the study area (unpublished data, based on our experience of conducting a large community-based trial in rural Bangladesh, ACTRN12612000588897), we require a sample of 900 pregnant women to retain 720 live births.

The estimated live births per cluster per month is approximately 19, based on the national rural crude birth rate of 23.3 per 1000 population..$^{28}$ Therefore, three months of recruitment will be sufficient to reach the required sample size. The estimated recruitment per cluster per month would be slightly more than eight.

\section{Data collection method and schedule}

At baseline, sociodemographic data and reproductive history will be collected from all pregnant women on enrolment. During the monthly follow-ups, information on health condition, current pregnancy status and healthcare services will be collected. We will conduct 
longitudinal dietary assessment for 4-7 non-consecutive days. All live newborn infants' birthweights will be assessed within 72 hours of birth with a hand-held spring balance scale (Salter scale with a capacity of five kg and an accuracy of $100 \mathrm{~g}$ ). Infants will wear only light clothes; the wrapping blanket will be taken off during weighing. The scale will be adjusted for the weight of the dress that the infant will be wearing before the process starts. SKs will collect the data during their regular scheduled visit to pregnant women and newborn children. A detailed schedule of assessment is shown in table 1.

Field supervisors will check $5 \%$ of the data randomly and notify a potential measurement problem. In addition, we will validate the dietary assessment data by repeating the assessment with independent interviewers in a subsample of participants (72; 36 each from intervention and control). Field supervisors, blinded to the outcome of interest, will also recapture $25 \%$ of the birthweight measures for data validity. The Salter scales used will be standardised every month. For institutional births, the recorded birthweights will be used.

Women will be designated as 'lost to follow-up' if they withdraw or move away and are untraceable for more than three consecutive visits. Information regarding the reasons for discontinuation will be recorded interviewing the relatives, neighbours or direct contact with the woman through mobile phones.

\section{Data management}

SKs will record the information in a paper book (one book for 25 participants), which will be transferred into an electronic file at the end of the intervention. The string variables will be coded with numeric values before inputting into Stata files. After completion of this process, the paper books will remain locked in the regional office of BRAC with access restricted to management staff only and will be destroyed after fouryears as per institutional guidelines. The research officer will de-identify the participants' data, code, clean and verify it (if needed). The principal investigator blinded to the trial arm will analyse the data.

\section{Statistical analysis}

All the women who are randomly assigned to either balanced plate or standard nutrition education will be analysed on an intention-to-treat basis. We will limit the birthweight data analysis to singleton liveborn infants, considering the strong relationship between multiple births and low birthweight. ${ }^{29}$ Dietary data will be analysed if we have at least two rounds of dietary assessment (baseline and follow-up), irrespective of pregnancy outcome.

The differences in performance of key cluster personnel (SKs) are expected to induce differences in response to the intervention. This makes the data hierarchical in nature due to the assumption of non-independence among those who live in the same cluster; hence we will use multilevel models to examine outcomes. Births being nested within clusters, we will use mixed effects regression models to measure the difference in effect (random intercept) between the treatment groups accounting for clusters and report the ICC. We will treat cluster as a random effect in the models, while the other explanatory variables will be treated as fixed effects. We will compare the distribution of prognostic factors between treatment groups and check for the effectiveness of randomisation. Any imbalance in the important prognostic factors will be adjusted by including them as covariates in the models.

We will use multivariable regression analysis to assess intervention effects holding other predictors constant, such as gestational age, infant sex, parity, age, socioeconomic status, education, the number of visits and time of the first visit after adjusting for the clusters. ${ }^{30}$ To assess the treatment effect on dietary caloric intake and diversity over time, we will use data from each visit distributed across subjects. We will compute inter-rater reliability to measure the level of agreement using ICC statistic among multiple coders (data collectors, SKs and field supervisors).$^{31}$ The analysis will be performed with Stata software (version 13.1).

\section{PROCESS EVALUATION}

We will apply the Trials of Improved Practices (TIPs) methodology to identify causes of any non-compliance of the advice provided to the women. We will purposively select subsamples (approximately 20) of the households, keeping the geographical and sociocultural (e.g., religion and economic status) diversities in mind. SKs will demonstrate the balanced plate to the pregnant women of these households, provide counselling to consume the balanced meal similar to the plate and ask them to continue this practice for 1 week. At the end of the week in a follow-up visit, the SKs will interview the women and identify significant barriers to incorporating the balanced diet into their dietary practices. The TIPs findings will provide feedback to adjust the design of the communication technique based on the practical experience of the trial participants. The feedback will be shared in the monthly meetings to adapt the content and strategy of the communication. Apart from the TIPs, we will assess the progress of the study against some verifiable indicators including the number of pregnant women enrolled and the number of nutrition messages delivered. Ticking the boxes through direct observations will check the number of nutrition messages delivered to the pregnant women. Any deviation from the target will be investigated for recovery actions. Any decline in performance against some verifiable indicators (pregnancy identification, ANC and postnatal visits) will suggest probable task overload of SKs. We will record all the barriers faced in the process of programme development and implementation. The process evaluation schedule is shown in table 1 . 


\section{DISCUSSION}

This paper describes a protocol for a two-arm cluster randomised controlled trial consisting of an intervention in which pregnant women receive nutrition education that includes a practical demonstration on preparing a nutritionally balanced meal. Nutrition education for pregnant women is understood to influence nutrition-related knowledge and dietary behaviour, pregnancy weight gain and birthweight. ${ }^{32}$

This new approach using a practical demonstration is a unique and promising method of behaviour change communication. This intervention is easy to implement by the existing community health workers and its participatory nature engages the pregnant women involved. It does not require any additional props or tools that are not readily available. Unlike typical nutrition education, our approach is more visual and interactive, which makes it easier for the participants to understand the messages clearly. Importantly, it does not require any food supplementation, which makes this intervention highly scalable and more likely to be sustainable in resource-poor settings including Bangladesh.

Nutrition education, which has been defined as 'any combination of educational strategies, accompanied by environmental supports, designed to facilitate voluntary adoption of food choices and other food and nutrition-related behaviours conducive to health and well-being...' has essentially three phases or components: motivational phase, action phase and environmental component. ${ }^{33}$ Our intervention addresses all the three components: verbal communication increases awareness and enhances motivation of the pregnant woman to adopt the intended behaviour; practical demonstration facilitates the ability to take action in practising a balanced meal; and communication with influential family members (husband, mother-in-law and mother) provides an enabling environment to support the woman's changed dietary behaviour. All of these three components are grounded in a health behaviour change communication theory, the 'Integrative Model of Behavioural Prediction'. According to this theory, a strong intention of a person to perform a behaviour, accompanied by the necessary skills and abilities to perform it, and a conducive environment results in the expected behaviour change. ${ }^{34}$

Using a practical demonstration in maternal nutrition education is a novel approach. However, this approach has been proved to be quite effective in other health education interventions. A study addressing oral health, provided a onehour lecture and practical demonstration to caregivers on oral hygiene, and measured the impact on oral infections (gingivitis and stomatitis). The results showed that gingivitis scores in the intervention group decreased to 0.28 units from a baseline of 1.37 , and the rate of stomatitis in the intervention decreased from $17 \%$ at baseline to $4 \% .{ }^{35}$ Gill and O'May investigated the utility of a practical pouring exercise to inform participants of their daily limits of alcohol consumption and found that almost half of the participants $(46 \%)$ were able to confirm exactly when they would exceed the limit. One-fifth of the participants thought that this practical pouring might influence their future alcohol drinking habit. ${ }^{36}$

Successful behaviour change is already observed in infant and young child feeding practices in Bangladesh with approaches like practical demonstration and counseling. ${ }^{37}$ An educational tool for practical demonstration (food plate and nutrition messages) to improve dietary diversity during pregnancy has been tested in one project in Bangladesh with the intention of improving maternal nutrition. ${ }^{38}$

\section{METHODOLOGICAL CONSIDERATIONS}

Contamination due to communication between the service providers cannot be completely ruled out as the study will be conducted in one geographic region. However, we will ensure that SKs from the intervention and control groups do not meet each other. These two groups will receive training separately and have monthly meetings in distinct groups. There is no chance of overlapping at the community level as $S K$ s work in distinct villages (villages are usually geographically separated by farm lands, canals and roads). Another limitation of the study is the multipurpose role of $S K s$ who will provide the 'balanced plate nutrition education' intervention and also act as data collectors. We anticipate that blinding to study hypothesis will reduce some of the measurement bias.

\section{ETHICS AND DISSEMINATION}

The present study was approved by the James P Grant School of Public Health, BRAC University Ethical Review Committee, Dhaka, Bangladesh.

We will communicate the final results to BRAC programme and research professionals, James P Grant School of Public Health professionals and students, public health academics, researchers and students at the University of Sydney and other relevant national and international forums. Research papers based on the study will be published in peer-reviewed journals. Everyone who makes a substantial contribution to the conduct of the project and/or to publications including conception, design, analyses and interpretation of data will be an author. We will acknowledge other contributions, such as project staff, BRAC management team, research assistants and community people via acknowledgement in the publication. Any researcher having a particular interest in the relevant field will be considered to get access to the full protocol and participant level dataset if felt crucial.

\section{TRIAL REGISTRATION}

The trial is registered in the Australian New Zealand Clinical Trials Registry (ANZCTR) on 25 January 2016. The registration number is ACTRN12616000080426. The protocol has been amended on 28 November 2016 with the designated Trial Registry. 


\section{DECLARATIONS}

\section{Consent to participate}

After pregnancy identification, informed consent will be obtained from all eligible participants before enrolling into the study. SKs will explain the nature and process of the study to the potential participants if they meet the inclusion criteria. For literate participants, the consent will be in a written form (a copy is attached in the Supplementary file 1) and for all others, it will be verbal. Participants will be assured about the confidentiality of data and will be allowed to withdraw at any time from the trial without any justification.

Acknowledgements We thank the Shasthya Kormis and Shasthya Shebikas of BRAC, and pregnant women and their families in Sherpur for their time in this study. The authors acknowledge the crucial role of the Sherpur BRAC Maternal Neonatal and Child Health (MNCH) project team and the team at BRAC headquarters. We gratefully acknowledge Dr Kaosar Afsana, Director, BRAC Health Nutrition and Population Programme for her tremendous support and guidance on the study implementation.

Contributors MC conceived the overall study and wrote the first draft of the protocol and this manuscript and CRG critically reviewed it. MJD provided critical input regarding study design, sample size calculation, and outcome evaluation and statistical analysis plan. AA provided crucial input on formative research and process evaluation design, and contributed to addressing the reviewers' comments and revision of the paper. MC and CRG obtained funding for the intervention. All authors critically reviewed and approved the final version of the manuscript and agree to be accountable for all investigations necessary to resolve questions related to accuracy or integrity of all or any part of the work.

\section{Competing interests None declared.}

Ethics approval James P Grant School of Public Health, BRAC University Ethical Review Committee.

Provenance and peer review Not commissioned; externally peer reviewed.

Open Access This is an Open Access article distributed in accordance with the Creative Commons Attribution Non Commercial (CC BY-NC 4.0) license, which permits others to distribute, remix, adapt, build upon this work non-commercially, and license their derivative works on different terms, provided the original work is properly cited and the use is non-commercial. See: http://creativecommons.org/ licenses/by-nc/4.0/

(C) Article author(s) (or their employer(s) unless otherwise stated in the text of the article) 2017. All rights reserved. No commercial use is permitted unless otherwise expressly granted.

\section{REFERENCES}

1. United Nations Children's Fund and World Health Organization. Low birthweight: country, regional and global estimates. New York: UNICEF, 2004.

2. Black RE, Allen LH, Bhutta ZA, et al. Maternal and child undernutrition: global and regional exposures and health consequences. Lancet 2008;371:243-60.

3. Gupta ND, Deding M, Lausten M. The effect of low birthweight on height, weight and behavioral outcomes in the medium-run. Econ Hum Biol 2013:11:42-55.

4. Victora CG, Adair L, Fall C, et al. Maternal and child undernutrition: consequences for adult health and human capital. Lancet 2008;371:340-57.

5. Goddeeris JH, Saigal S, Boyle MH, et al. Economic outcomes in young adulthood for extremely low birthweight survivors. Pediatrics 2010;126:e1102-e1108.

6. Triunfo S, Lanzone A. Impact of maternal under nutrition on obstetric outcomes. J Endocrinol Invest 2015;38:31-8.

7. Smith $\mathrm{CA}$. undefined Effects ofmaternal under nutrition upon the newborn infant in Holland (1944-1945). The Journal of pediatrics 1947;30:229-43.

8. AkitakeY, KatsuragiS, HosokawaM et al, Moderate maternal food restriction in mice impairs physical growth, behavior, and neurodevelopment of offspringNutrition Research 3517687 2015;:--
9. KramerMS . Determinants of low birth weight: methodological assessment and meta-analysis Bulletin of the World Health Organization 655663737.1987

10 National Institute of Population Research and Training (NIPORT), Mitra and Associates, and ICF International. 2013. Bangladesh Demographic and Health Survey 2011. Dhaka, Bangladesh and Calverton, Maryland, USA: NIPORT, Mitra and Associates, and ICF International.

11 Alam DS, Van Raaij JM, Hautvast JG, et al. Energy stress during pregnancy and lactation: consequences for maternal nutrition in rural Bangladesh. Eur J Clin Nutr 2003;57:151-6.

12 National Institute of Population Research and Training (NIPORT), Mitra and Associates, ICF International. Bangladesh Demographic and Health Survey 2014. Dhaka, Bangladesh and Rockville, Maryland, USA: NIPORT, Mitra and Associates and ICF International, 2016.

13 Islam MZ, Akhtaruzzaman M, Lamberg-Allardt C. Nutritional status of women in Bangladesh: comparison of energy intake and nutritional status of a low income rural group with a high income urban group. Asia Pac J Clin Nutr 2004;13:61-8.

14 Alam DS, Van Raaij JM, Hautvast JG, et al. Energy stress during pregnancy and lactation: consequences for maternal nutrition in rural Bangladesh. Eur J Clin Nutr 2003;57:151-6.

$15 \mathrm{FAO}$ and FHI 360 . Minimum dietary diversity for women: a guide for measurement. Rome: FAO, 2016.

16 Arimond M, Torheim LE, Wiesmann D. Dietary diversity as a measure of the micronutrient adequacy of women's diets: results from rural Bangladesh site. Washington, DC: Food and Nutrition Technical Assistance II Project (FANTA-2), FHI 360, 2009.

17 Gluckman PD, Sizonenko SV, Bassett NS. The transition from fetus to neonate--an endocrine perspective. Acta Paediatr Suppl 1999;88:5-11.

18 Tabrizi FM, Saraswathi G. Maternal nutrient intake and maternal serum micronutrients and their relation to birthweight: a longitudinal study. Int J Collab Res Intern Med Publ Health 2011;3:617-32.

19 Widga AC, Lewis NM. Defined, in-home, prenatal nutrition intervention for low-income women. J Am Diet Assoc 1999;99:1058-62.

20 Ota E, Hori H, Mori R, et al; Antenatal dietary education and supplementation to increase energy and protein intake (review). John Wiley \& Sons: Cochrane Database of Systematic Reviews, 2015.

21 Victora CG, Barros FC, Assunção MC, et al. Scaling up maternal nutrition programs to improve birth outcomes: a review of implementation issues. Food Nutr Bull 2012;33(2 Suppl):S6-S26.

22 Monitoring the Situation of Children and Women Project. National low birthweight survey of Bangladesh, 2003-2004. Dhaka: Bangladesh Bureau of Statistics, 2005.

23 Klemm RD, Merrill RD, Wu L, et al. Low-birthweight rates higher among Bangladeshi neonates measured during active birth surveillance compared to national survey data. Matern Child Nutr 2015;11:583-94.

24 West KP, Shamim AA, Mehra S, et al. Effect of maternal multiple micronutrient vs iron-folic acid supplementation on infant mortality and adverse birth outcomes in rural Bangladesh: the JiVitA-3 randomized trial. JAMA 2014;312:2649-58.

25 United Nations General Assembly. 2014. Report of the Open Working Group of the General Assembly on Sustainable Development Goals.

26 ProPAN: Process for the Promotion of Child Feeding. Washington, DC: PAHO, 2013.

27 District Statistics 2011: Sherpur. Bangladesh Bureau of Statistics (BBS) Statistics andlnformatics Division (SID), Ministry of Planning, Government of the People's Republic of Bangladesh, 2013.

28 Population and Housing Census 2011. Socio-Economic and Demographic Report: Bangladesh Bureau of Statistics (BBS) Statistics and Informatics Division (SID), Ministry of Planning, 2012.

29 Nahar Q, Choudhury S, Faruque MO, et al. Desirable Dietary Pattern for Bangladesh. National Food Policy Capacity Strengthening Programme, 2013.

30 Shaheen N, Rahim ATM, Mohiduzzaman M, et al. Food Composition Table for Bangladesh. Institute of Nutrition and Food Science, Centre for Advanced Research in Sciences, University of Dhaka, 2013.

31 Hayes RJ, Moulton LH. Cluster randomised trials. Boca Raton: CRC Press, 2009.

32 Zeng L, Dibley MJ, Cheng Y, et al. Impact of micronutrient supplementation during pregnancy on birthweight, duration of gestation, and perinatal mortality in rural western China: double blind cluster randomised controlled trial. BMJ 2008;337:a2001-15.

33 Frenkel H, Harvey I, Newcombe RG. Improving oral health in institutionalised elderly people by educating caregivers: a 
randomised controlled trial. Community Dent Oral Epidemiol 2001;29:289-97.

34 Beiguelman B, Colletto GMDD, Franchi-Pinto C, et al. Santa Casa de Misericórdia de São Paulo. Birthweight of twins: 1. The fetal growth patterns of twins and singletons. Genet Mol Biol 1998;21:151-4.

35 Lee J. Long-term effects of low birthweight. Agricultural Res 1999;47:10.

36 Hallgren KA. Computing inter-rater reliability for observational data: an overview and tutorial. Tutor Quant Methods Psychol 2012;8:23-34.

37 Akter SM, Roy SK, Thakur SK, et al. Effects of third trimester counseling on pregnancy weight gain, birthweight, and breastfeeding among urban poor women in Bangladesh. Food Nutr Bull 2012;33:194-201.

38 Contento IR. Nutrition education: linking research, theory, and practice. Asia Pac J Clin Nutr 2008;17 Suppl 1:176-9.
39 Fishbein M, Yzer MC. Using Theory to Design Effective Health Behavior Interventions. Communication Theory. 2003;13:164-83.

40 Gill J, O'May F. Practical demonstration of personal daily consumption limits: a useful intervention tool to promote responsible drinking among UK adults? Alcohol Alcohol 2007;42:436-41.

41 International Food Policy Research Institute. Rapid improvements in infant and young child feeding practices resulting from a large-scale program: Alive \& Thrive's approach and results in Bangladesh. 2015.

42 Shamim AA, Tegenfeldt K, Aradhya K, et al. Designing a food plate for dietary counselling of pregnant women in Bangladesh. ENN: SHIKHA project, 2016.

43 National Institute of Population Research and Training (NIPORT), Mitra and Associates, and ICF International. Bangladesh Demographic and Health Survey 2011. Dhaka, Bangladesh and Calverton, Maryland, USA: NIPORT, Mitra and Associates, and ICF International, 2013.1999;88:5-11;88:5-11. 